Artículo científico

Volumen 32(2):573-586. Mayo-agosto, 2021

e-ISSN 2215-3608, doi:10.15517/am.v32i2.39673

https://www.revistas.ucr.ac.cr/index.php/agromeso

\title{
Factibilidad de la producción de huevos de gallinas ponedoras con acceso a pastoreo $^{1}$
}

\section{Egg production feasibility in laying hen with access to grazing}

\author{
Ana Cruz-Bermúdez ${ }^{2}$,Rodolfo WingChing-Jones ${ }^{3}$,Rebeca Zamora-Sanabria ${ }^{3}$
}

1 Recepción: 25 de noviembre, 2019. Aceptación: 28 de octubre, 2020. Este trabajo formó parte del trabajo de Licenciatura de Ingeniería Agronómica con énfasis en Zootecnia de la primera autora, financiada por la Universidad de Costa Rica, San José, Costa Rica.

2 Universidad de Costa Rica, Escuela de Zootecnia, San José Costa Rica. ancruzber@hotmail.com (https://orcid.org/0000-0002-1940-0819).

3 Universidad de Costa Rica, Escuela de Zootecnia, Centro de Investigación en Nutrición Animal (CINA), San José, Costa Rica. rodolfo.wingching@ucr.ac.cr (autor para correspondencia, https://orcid.org/0000-0002-8009-2210); rebeca.zamora@ucr.ac.cr (https://orcid.org/00000002-9679-4647).

\section{Resumen}

Introducción. La producción de gallinas con acceso a pastoreo integra el manejo del ave, forraje y la comercialización. Objetivo. Describir los sistemas de producción de gallinas ponedoras con acceso a pastoreo en Costa Rica y determinar la factibilidad de la implementación de un modelo de producción. Materiales y Métodos. Durante los años 2017 y 2018, se recolectó y analizó la información de producción, sanidad, pastoreo y manejo del huevo en seis granjas con aves de postura que presentan acceso al pastoreo. Para determinar la factibilidad del modelo de producción propuesto, se elaboró un flujo de caja (diez años), que contempló las inversiones para el desarrollo del sistema y una tasa de inflación del $10 \%$ a través de los años. Se calcularon los costos de inversión, operación e ingresos por venta de huevo, gallinas de descarte y gallinaza. Se calcularon el VAN, TIR, relación B/C y punto de equilibrio. Resultados. El $66 \%$ de los sistemas manejan aves especializadas, densidades entre 5 a 12 y 1 a 3 aves $\mathrm{m}^{-2}$ en el galpón y área de pastoreo respectivamente. El pastoreo se inició entre las 22 y 32 semanas, con sesiones de 2 a 10 horas día ${ }^{-1}$. Cinco sistemas no sustituyeron el alimento por forraje, lo consideran como aporte adicional. La propuesta, es demandante en instalaciones, terreno y equipo de trabajo. El alimento representó el $41 \%$ de los costos operativos, seguido por el personal y la compra de las aves. Es rentable, al obtener un VAN positivo $(\$ 31455,39)$, TIR de 21,48 $\%$, una relación $\mathrm{B} / \mathrm{C}>1$ y se recupera la inversión a los seis años. Conclusión. Las granjas con gallinas ponedoras con acceso a pastoreo podrían mejorar en aspectos nutricionales y del cultivo del forraje. Además, son sistemas de producción que presentan indicadores económicos que propician su implementación.

Palabras clave: producción animal, diagnóstico, bienestar animal, avicultura.

\begin{abstract}
Introduction. The production of hens with access to grazing integrates bird, fodder, and marketing management. Objective. To describe laying hens' production systems with grazing access in Costa Rica and determine the feasibility of implementing aproduction model. Materials and methods. During the years 2017 and 2018, information on the
\end{abstract}


production, health, grazing, and egg management was collected and analyzed in six farms with laying birds that have access to grazing. To determine the feasibility of the proposed production model, a cash flow (ten years) was prepared, which included the investments to develop the system, and an inflation rate of $10 \%$ through the years. The costs of investment, operation, and income from the sale of the eggs, discarded hens, and poultry litter were analyzed. The NPV, IRR, C/B ratio, and break-even point were calculated. Results. Sixty six percent of the systems use specialized birds, densities between 5 to 12 , and 1 to 3 birds $\mathrm{m}^{-2}$ in the shed and grazing area, respectively. Grazing began at 22 and 32 weeks, with $2-10 \mathrm{~h} \mathrm{day}^{-1}$. Five systems did not substitute feed for fodder; they consider it as an added value. The proposal is demanding in facilities, plot, and work equipment. Feeds represent $41 \%$ of the operational costs, followed by labor (22\%), and bird purchase (17\%). It is profitable, by obtaining a positive NPV (\$31 455.39) and IRR of 21.48 $\%$ and the $\mathrm{C} / \mathrm{B}$ ratio $>1$, and the investment will be recovered after six years. Conclusions. Farms with laying hens with access to grazing evaluated in this work could improve in nutritional aspects and forage cultivation. In addition, they are production systems that present economic indicators that favor their implementation.

Keywords: animal production, diagnosis, animal welfare, poultry.

\section{Introducción}

En el año 2017 la avicultura representó el $24 \%$ de las actividades primarias del sector pecuario y el 5,4 $\%$ del valor bruto de la producción agropecuaria en Costa Rica (Secretaria Ejecutiva de Planificación Sectorial Agropecuaria, 2018). En el caso de la industria del huevo en Costa Rica, del año 2012 al 2016, la cantidad de gallinas ponedoras aumentó de 2,89 a 3,80 millones, y el consumo per cápita de huevo pasó de 200 a 206 unidades por persona, cifra que supera a países centroamericanos y a Brasil (Ruiz, 2017). De la población nacional de gallinas, el $63 \%$ se encuentran confinadas en jaulas con una densidad de $450-510 \mathrm{~cm}^{2}$ por ave en sistemas intensivos (Monge, 2016), la restante se mantiene en sistemas en piso, mientras que la cantidad de sistemas alternativos y de producción de huevo con acceso a pastoreo no se encuentran registrados en el país.

El concepto de producción de huevos de gallinas con acceso a pastoreo, se asocia al sistema de alojamiento, que facilita a las aves desplazarse de forma libre en un área mayor con el suelo cubierto de forraje y que les permite expresar comportamientos naturales (Salatin, 1996). Manejo que les da la posibilidad de consumir forraje, insectos, utilizar vitaminas naturales, una mayor tasa de recambio de aire y luz natural (Campbell et al., 2017). Recordando que el sistema digestivo que presentan las aves no las deja aprovechar la fibra, por lo que el material consumido durante el periodo de acceso al pastoreo se considera como nutrimentos adicionales a los provenientes del consumo de alimento balanceado establecido para cada raza o línea de ave utilizada (Singh \& Cowieson, 2013).

En Costa Rica, para el año 2018, no existía legislación para la producción de huevos bajo la modalidad de acceso a pastoreo, ni para la implementación o supervisión de otros manejos alternativos de producción; sin embargo, los consumidores se interesan cada vez más por la adquisición de productos avícolas producidos bajo modalidades alternativas a las convencionales.

El cumplir con los requerimientos nutricionales de las aves con alimento balanceado y realizar un adecuado manejo del cultivo del forraje, es la base de los sistemas de aves con acceso a pastoreo, sin dejar de lado la parte sanitaria y la bioseguridad del sistema (Chaves, 2011). La aplicación de vacunas dependerá de la presencia de patógenos en la granja y de la zona geográfica; sumado a la implementación de prácticas diarias de limpieza del equipo e instalaciones (Lohmann Tierzucht, 2013), aspersiones con desinfectante al ambiente interno del galpón (Kahrs, 1995), aspersiones de las instalaciones contra parásitos externos (Axtell \& Arends, 1990), control de roedores (Bell \& Weaver, 2002) y medicación con mucolíticos cuando amerite (Carrillo et al., 2016), permiten 
optimizar los indicadores productivos y la salud de las aves (Dhama et al, 2015). Debido a que las aves en pastoreo están más expuestas al contacto con aves silvestres, plagas, vectores y depredadores, se recomienda realizar una vigilancia estricta y frecuente de las enfermedades exóticas en el país como influenza aviar y la enfermedad de Newcastle y mantener estrictas medidas de bioseguridad dentro de las instalaciones y las que sean posibles de realizar fuera de las galeras.

La percepción de los consumidores es que las gallinas que tienen oportunidad de salir de las instalaciones y tener contacto con aire fresco producen huevos "más naturales", bajos en grasa y altos en proteínas y vitaminas (Miao et al., 2005) y el acceso a áreas abiertas les proporciona mayor bienestar (Campbell et al., 2017). Por esta razón, los productores al observar este nicho de mercado, con más frecuencia implementan o adaptan sus producciones para satisfacer esta demanda. Para el año 2012, se registraron cuatro empresas, de amplia trayectoria avícola en Costa Rica, que incursionaron en este mercado de huevo. En este mismo año, el $2 \%$ de la población de aves de una de las empresas, se encontraba bajo el sistema de producción con acceso a pastoreo (Brenes, 2012). Además, en uno de los puestos de venta de huevos consultado en el año 2012, el $11 \%$ del total de huevo comercializado provenía de sistemas con pastoreo de aves, con un sobreprecio del $30 \%$ por $\mathrm{kg}\left(\phi 2414 \mathrm{~kg}^{-1}, \$ 1=\phi 593\right)$, al compararse con el huevo proveniente de producciones convencionales (Brenes, 2012).

Los sistemas alternativos de manejo de aves de postura inciden en la rentabilidad y la calidad del producto final. En México, según la percepción general del sector, el costo de producción puede llegar a duplicarse y puede impactar hasta $40 \%$ del precio final del huevo. Por otro lado, los cambios de una producción convencional a una alternativa, causaron aumentos en los costos de producción en alrededor del $40 \%$ por docena producida en el Estado de California en Estados Unidos (Sumner et al., 2011). También, un factor adicional que eleva el costo de producción es el incremento en el consumo de alimento por el desgaste energético que tiene el ave al desplazarse, siendo siempre el costo de alimentación el más alto en cualquiera de los dos sistemas (Muñoz, 2016). El costo de transición en la infraestructura del sistema convencional al de acceso a pastoreo puede variar, pero se estima que el gasto adicional es de $15 \%$, el cual incluye el costo de la tierra y la mano de obra adicional (Miao et al., 2005). Como resultado, los huevos producidos en sistemas sin jaulas presentan un precio premium. En enero del 2016, el precio al por mayor en Estados Unidos fue de $\$ 0,52$ por docena más alto que los huevos convencionales (Sumner et al., 2011).

El objetivo de este trabajo fue describir los sistemas de producción de gallinas ponedoras con acceso a pastoreo en Costa Rica y determinar la factibilidad de la implementación de un modelo de producción.

\section{Materiales y métodos}

En una primera etapa se recolectó y analizó la información disponible en seis granjas con aves de postura que presentaron acceso al pastoreo, para conocer la forma en que se conceptualiza este sistema alternativo en Costa Rica. Con base en esta información se determinó la factibilidad de la implementación de un modelo de producción de huevo con gallinas ponedoras con acceso a pastoreo en condiciones del trópico.

\section{Diagnóstico productivo}

Se realizó un diagnóstico para conocer las características y componentes que presentaban los sistemas de producción de huevo con acceso a pastoreo que se encontraban en funcionamiento en Costa Rica. Para tal fin, se visitaron seis granjas ubicadas en San José, Cartago, Coronado, Atenas, Esparza y San Carlos, durante el segundo semestre del año 2017 y el primer semestre del 2018. La recopilación de los datos se realizó mediante una herramienta tipo encuesta, que permitió reflejar las condiciones de producción de cada sistema. Se registró y analizó la información relacionada con la densidad de aves, estirpe utilizada, alimentación, manejo sanitario, manejo de las instalaciones y de las aves, las áreas de pastoreo, manejo del huevo, producción y comercialización. 


\section{Descripción del modelo de producción propuesto}

\section{Instalaciones y equipo}

El modelo de producción propuesto requiere la construcción de un galpón para mantener las gallinas ponedoras con acceso a pastoreo, el cual se ubica en el módulo aviar de la Sede del Atlántico de la Universidad de Costa Rica,

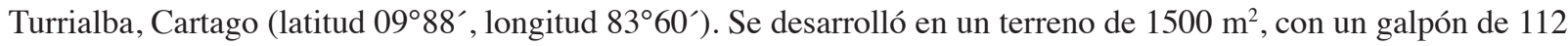
$\mathrm{m}^{2}$ con capacidad para 1000 aves y con tres áreas de pastoreo o parques de $300 \mathrm{~m}^{2}$ cada una, con una densidad de 9 y 3 aves $\mathrm{m}^{-2}$, respectivamente. El galpón se diseñó con equipo automatizado para los comederos, bebederos, iluminación y ventilación, además de bodegas de almacenamiento de huevo, alimento balanceado y utensilios, y vestidores. La postura se propuso en nidos individuales y la recolección de los huevos fue manual.

\section{Indicadores productivos y comercialización}

Por su potencial genético, las aves del modelo propuesto fueron de la línea genética Isa Brown, las cuales se deben de comprar en etapa de pre-postura, alrededor de las diecisiete semanas de edad, finalizando el ciclo productivo a las ochenta semanas de edad. La compra de las aves se estimó en base a los diez años proyectados para la investigación, a excepción de los años tres, seis y nueve (al considerar 52 semanas por año), ya que las aves se encontrarían aún en periodo de producción (ochenta semanas). Se ofreció a las aves una cantidad promedio de 115 g de alimento balanceado en dos fases: fase I (18\%PC, 2900 kcal energía metabolizable) y fase II (17 \%PC y 2750 kcal energía metabolizable). El manejo sanitario incluyó la desparasitación, aspersiones con yodo y la limpieza y desinfección del equipo e instalaciones. Se utilizaron valores promedio para los indicadores productivos, en el caso de la producción de huevos se consideró $84 \%$ de postura, un peso de huevo de 62 g y una sobrevivencia de $94 \%$. La comercialización de los huevos se desarrolló en la misma granja, en grupos de treinta unidades colocadas en cartones específicos para almacenar huevo, con envoltura de plástico y etiquetas que indicaba el alojamiento de las aves con acceso a pastoreo. El precio promedio al que se comercializó el kg de huevo fue de $\phi 2000(\$ 1=\varnothing 611$ colones netos).

\section{Análisis económico}

La recopilación de información se hizo a través de consultas a tipologías constructivas del Ministerio de Hacienda, comercios, profesionales en economía agrícola y en trámites de crédito y avalúos, permitió realizar la estructura general del sistema de producción de gallina ponedora con acceso a pastoreo. Se elaboró un flujo de caja proyectado a diez años, el cual estuvo afectado por una tasa de inflación del $10 \%$ a través de los años, donde se contemplaron las inversiones necesarias para el desarrollo del sistema (año 0) y para los demás años. Con lo anterior, se calcularon los costos de inversión y de operación (fijos y variables) para el establecimiento de dicha producción, así como los ingresos provenientes de la venta de huevo, gallinas de descarte y gallinaza. Se determinó la rentabilidad del sistema a partir de los factores de valor actual neto (VAN), tasa interna de retorno (TIR), relación beneficio/costo (B/C) y punto de equilibrio (Sapag \& Sapag, 2008). Se sensibilizaron las variables de porcentaje de postura, precio del kilogramo de huevo y el precio del $\mathrm{kg}$ de alimento balanceado, los cuales incidieron de forma directa sobre el VAN y el TIR. Para el desarrollo del proyecto, se consideró el financiamiento completo en entidades bancarias, con una tasa de interés anual del $10 \%$ fijo, por un plazo de diez años. Para efectos del VAN, se asumió una tasa de rendimiento anual del $14 \%$, la cual es la mínima para que el inversionista decida realizar el negocio. 


\section{Resultados}

\section{Diagnóstico productivo}

El $66 \%$ de las granjas visitadas manejaban aves especializadas en producción de huevos, como la Isa Brown (50 $\%)$ y la Hy-line (50 \%). Dos granjas de pastoreo tenían gallinas del cruce Sex Link Negro y, por último, la Rhode Island Red. Las densidades de estas aves fluctuaron entre cinco y doce aves por metro cuadrado en los galpones, según las características del sistema (Cuadro 1). Mientras que en los parques de pastoreo o áreas de pastoreo, las densidades variaron entre 1 y 3 aves $\mathrm{m}^{-2}$, excluyendo el sistema 2 que ofreció $0,02 \mathrm{~m}^{2}$ por ave al día (Cuadro 1).

Cuadro 1. Características generales de la producción de gallinas ponedoras con acceso a pastoreo en seis granjas en Costa Rica. 2017. Table 1. General characteristics of the production of laying hens with access to grazing in six farms in Costa Rica. 2017.

\begin{tabular}{|c|c|c|c|c|c|c|c|c|c|c|c|}
\hline \multirow[t]{2}{*}{ Granja } & \multicolumn{2}{|c|}{$\begin{array}{l}\text { Densidad } \\
\left({\left.\text { aves } \mathbf{m}^{-2}\right)}^{-}\right.\end{array}$} & \multirow[t]{2}{*}{ Estirpe } & \multirow[t]{2}{*}{ Alimentación } & \multirow{2}{*}{$\begin{array}{c}\text { Tipo de } \\
\text { forraje en } \\
\text { parques }\end{array}$} & \multirow{2}{*}{$\begin{array}{l}\mathrm{N}^{\circ} \text { de } \\
\text { par- } \\
\text { ques }\end{array}$} & \multirow{2}{*}{$\begin{array}{c}\text { Edad al } \\
\text { salir a } \\
\text { pastoreo } \\
\text { (semanas) }\end{array}$} & \multirow{2}{*}{$\begin{array}{l}\text { Tiempo } \\
\text { en pasto- } \\
\text { reo al día } \\
\text { (horas) } \\
\end{array}$} & \multirow{2}{*}{$\begin{array}{c}\mathbf{N}^{\circ} \\
\text { salidas } \\
\text { hacia } \\
\text { parques }\end{array}$} & \multirow{2}{*}{$\begin{array}{c}\text { Manejo } \\
\text { de pas- } \\
\text { tura }\end{array}$} & \multirow{2}{*}{$\begin{array}{c}\text { Afeccio- } \\
\text { nes en } \\
\text { las aves }\end{array}$} \\
\hline & Galeras & Parques & & & & & & & & & \\
\hline 1 & 6 & 0,3 & Sex Link & $\begin{array}{l}\text { Alimento } 18 \\
\% \mathrm{PC}^{* *} .85 \mathrm{~g} \\
\text { ave }^{-1} \text { con una } \\
\text { sustitución } \\
\text { de alrededor } \\
\text { del } 30-40 \% \text {. } \\
\text { Ofrecimiento } \\
\text { de hojas de } \\
\text { brócoli y kale }\end{array}$ & Kikuyo & 3 & 22 & 4,5 & 1 & N/A* & $\begin{array}{l}\text { Pará- } \\
\text { sitos, } \\
\text { picaje y } \\
\text { caniba- } \\
\text { lismo }\end{array}$ \\
\hline 2 & 10 & 39 & Isa Brown & $\begin{array}{l}\text { Diferentes fa- } \\
\text { ses de alimen- } \\
\text { to balanceado } \\
\text { comercial. No } \\
\text { realizan susti- } \\
\text { tución }\end{array}$ & $\begin{array}{l}\text { Combi- } \\
\text { nación de } \\
\text { Brizantha, } \\
\text { Brachia- } \\
\text { rias }\end{array}$ & 7 & 30 & 2 & 1 & Corta & $\begin{array}{c}\text { Depreda- } \\
\text { dores }\end{array}$ \\
\hline 3 & 5 & 1,25 & Isa Brown & $\begin{array}{l}\text { Diferentes fa- } \\
\text { ses de alimen- } \\
\text { to balanceado } \\
\text { comercial. No } \\
\text { realizan susti- } \\
\text { tución }\end{array}$ & $\begin{array}{l}\text { Estrella, } \\
\text { natural, } \\
\text { maní fo- } \\
\text { rrajero }\end{array}$ & 3 & 29 & 8 & $6-10$ & N/A & Parásitos \\
\hline 4 & 7 & 1 & Hy-line & $\begin{array}{l}\text { Diferentes fa- } \\
\text { ses de alimen- } \\
\text { to balanceado } \\
\text { comercial. No } \\
\text { realizan susti- } \\
\quad \text { tución }\end{array}$ & $\begin{array}{c}\text { Axonopus } \\
\text { compres- } \\
\text { sus }\end{array}$ & 1 & 25 & 8 & 12 & Corta & Parásitos \\
\hline 5 & 12 & 1 & Hy-line & $\begin{array}{l}\text { Alimento } 18 \% \\
\text { PC } 120 \mathrm{~g}^{-1} \text {. } \\
\text { No realizan } \\
\text { sustitución }\end{array}$ & $\begin{array}{l}\text { Axonopus } \\
\text { compres- } \\
\text { sus }\end{array}$ & 3 & 25 & 10 & 1 & $\begin{array}{l}\text { Ferti- } \\
\text { rriego }\end{array}$ & Parásitos \\
\hline 6 & 6 & 3 & $\begin{array}{l}\text { Sex Link } \\
\text { Negro, } \\
\text { Rhode Is- } \\
\text { land Red }\end{array}$ & $\begin{array}{l}\text { Alimento } 18 \% \\
\text { PC. } 135 \mathrm{~g} \text { ave } \\
\text { No realizan } \\
\text { sustitución }\end{array}$ & Estrella & $\begin{array}{c}3 \\
\text { cada } \\
\text { lote }\end{array}$ & 32 & 6 & 3 & Corta & $\begin{array}{l}\text { Pará- } \\
\text { sitos, } \\
\text { picaje y } \\
\text { caniba- } \\
\text { lismo }\end{array}$ \\
\hline
\end{tabular}

*N/A= no aplica; **PC= proteína cruda en base seca. / N/A= not applicable; **PC= crude protein on a dry basis. 
La especie forrajera presente en las granjas se asoció a la ubicación del sistema de producción, donde se encontró el uso del pasto Kikuyo (Kikuyocloa clandestinus), estrella africana (Cynodon nlemfluensis), natural (Axonopus compresus) y brizantha (Brachiaria brizantha). Se observó un desconocimiento en el manejo del componente forrajero y del pastoreo de las aves, donde la constante fue un deterioro de la pastura. La práctica de manejo más recurrente fue la corta de forraje posterior a la salida de las aves para uniformizar el parque. Las aves iniciaron su primer pastoreo cuando presentaron edades entre 22 y 32 semanas, con sesiones de 2 hasta $10 \mathrm{~h}$ al día. Con el inconveniente de que los galpones presentaban pocos accesos a los parques, lo que limitó la oportunidad de que todas las aves tuvieran acceso al pastoreo. Según las condiciones del clima, las aves en periodos de pastoreo se agrupaban en el primer tercio del parque cercano al galpón, comportamiento que se observó en forma generalizada en los seis sistemas visitados.

En aspectos nutricionales, cinco de las seis granjas no realizaron sustitución de alimento balanceado por forraje, con base en lo consumido durante el pastoreo, considerándolo un aporte adicional de nutrimentos. Además, el $50 \%$ utilizaron diferentes fases de alimentación en el ciclo productivo, lo que benefició el bienestar de las aves al asegurar los requerimientos nutricionales. Solo en una granja se registró una sustitución entre el 30 al $40 \%$ del alimento requerido, lo que provocó un comportamiento voraz y agresivo de las aves, el cual se exacerbaba cuando se ofrecía hojas de brócoli (Brassica oleracea var. Itálica) y kale (Brassica oleracea var. Sabellica). La suplementación de carbonato de calcio como grit (piedritas insolubles), fue una práctica encontrada en todos los sistemas, con la intención de mejorar la calidad de la cáscara de los huevos de lotes de gallinas viejas, la cual pierde su grosor conforme aumenta la edad de las aves.

Se observó que la mayoría de los problemas sanitarios que presentaron las aves en las granjas visitadas fueron parasitismo (83\%), picaje (33\%), desplume (pérdida de plumas alrededor de la cola y en el dorso) (33\%) y pérdidas por depredación de animales silvestres como serpientes, coyotes y perros (17\%). En general, el porcentaje de mortalidad se mantuvo dentro de los parámetros indicados por las casas genéticas. Otros problemas registrados en estas granjas, que no se relacionaron con el sistema alternativo, fueron compromisos en la integridad intestinal de las gallinas y errores en la aplicación de vacunas contra enfermedades respiratorias.

En el caso de los nidos, en su mayoría eran de metal para aves reproductoras, pero también se presentaron adaptaciones de recipientes plásticos (capacidad de cinco galones) y de madera con divisiones. En el caso del material utilizado como cama, estos variaron entre cascarilla de arroz y heno, y su cambio se realizaba cada quince días. Para la recolección de los huevos, la frecuencia fue de dos hasta seis veces al día, de forma manual, y dependiente del tamaño de la explotación. Para la recolección, tres granjas utilizaban la ayuda de cajas con un riel, mientras que, en los demás sistemas, los huevos fueron depositados en cubetas plásticas. Todas las granjas registraron problemas de huevo puesto en el piso del galpón. También se registraron porcentajes de huevo sucio y quebrado, pero no dispusieron de la información de la cantidad exacta. Los huevos sucios se limpiaron con lijas y trapos mojados y se comercializaron como huevos de segunda categoría.

\section{Inversiones y estructura de costos de operación del modelo desarrollado}

El modelo propuesto fue demandante en espacio físico e instalaciones, donde este rubro alcanzó el $80 \%$ de la inversión inicial, seguido del terreno $(13,17 \%)$ y equipo de trabajo $(5,5 \%)$ (Cuadro 2). Las instalaciones y el equipo se calcularon de acuerdo con las necesidades de espacio y número de aves, donde los costos se determinaron

a partir de valores promedio de mercado. Se requeriría de un terreno de $1500 \mathrm{~m}^{2}$ como mínimo, distribuido en 164 $\mathrm{m}^{2}$ de construcción y $900 \mathrm{~m}^{2}$ de áreas de pastoreo, distribuidas en tres parques $\left(300 \mathrm{~m}^{2}\right)$. 
Cuadro 2. Inversiones iniciales, vida útil y depreciaciones de los insumos requeridos para establecer la infraestructura, equipo y terreno para la producción de gallinas ponedoras con acceso a pastoreo. Cartago, Costa Rica. 2017.

Table 2. Initial investments, useful life, and depreciation of the required inputs to establish the infrastructure, equipment, and land for the production of laying hens with access to grazing. Cartago, Costa Rica. 2017.

\begin{tabular}{lcccc}
\hline Rubro & Inversión (\$) & Porcentaje (\%) & Vida útil (años) & Depreciación anual (\$) \\
\hline $\begin{array}{l}\text { Instalaciones y áreas de } \\
\text { pastoreo }\end{array}$ & 49656,30 & 80 & 50 & 993,13 \\
Terreno & 8183,31 & 14 & N/A & \\
Equipo & 3436,99 & 6 & 10 & 294,60 \\
Total & 62094,93 & 100 & & 1446,81 \\
\hline
\end{tabular}

Fuente / Source: Ministerio de Hacienda $(2015)^{4}$, Cruz (2018) ${ }^{5}$, Piedra (2018) ${ }^{6}$, Suministro Avícola Belén (2018)7.

\section{Costos de operación}

El rubro con mayor porcentaje a lo largo de los años evaluados, fue el alimento balanceado, el cual representó el $41 \%$ del total, seguido de $22 \%$ de pago de la mano de obra y $17 \%$ de compra de las aves en período de prepostura (Cuadro 3).

Cuadro 3. Estructura y valor de costos operativos en los años 1, 5 y 10 para el establecimiento de un sistema productivo de gallina ponedora con acceso a pastoreo. Cartago, Costa Rica. 2017.

Table 3. Structure and operational costs value in years 1, 5, and 10 for the establishment of a productive system of laying hens with access to grazing. Cartago, Costa Rica. 2017.

\begin{tabular}{lccc}
\hline Insumo & Año 1 (\$) & Año 5 (\$) & Año 10 (\$) \\
\hline Alimento balanceado & 18115,59 & 26523,03 & 42715,61 \\
Compra de aves & 7446,81 & 10812,77 & 17127,42 \\
Material de la cama & 81,83 & 11981,00 & 191,36 \\
Mantenimiento de instalaciones & 1047,46 & 1533,59 & 2469,86 \\
y equipo & & & 302,94 \\
Manejo sanitario & 128,48 & 188,10 & 12347,31 \\
Depreciaciones & 6124,06 & 8287,89 & 22576,09 \\
Mano de obra & 9574,47 & 14017,98 & 5487,03 \\
Cargas sociales & 2327,04 & 3407,02 & 5371,95 \\
Agua/electricidad/teléfono & 2278,23 & 3335,56 & 463,10 \\
Impuestos municipales & 196,40 & 287,55 & 1513,28 \\
Empaque de huevo & 1513,28 & 2215,59 & 32,73 \\
Uniformes & 32,73 & 32,73 & 2510,03 \\
Imprevistos 2 \% & 1374,73 & 1782,83 & 120560,12 \\
Total costos operativos & 60176,30 & 81734,50 & \\
\hline
\end{tabular}

\footnotetext{
${ }^{4}$ Manual de Valores Base Unitarios por Tipología Constructiva del Ministerio de Hacienda (2015).

5 R. Cruz (2018). Perito externo del Banco Nacional de Costa Rica. Comunicación personal. Cartago, Costa Rica.

${ }^{6}$ Suministro Avícola Belén. (2018). Comunicación personal. San José, Costa Rica.

7 M. Piedra (2018). Perito externo del Banco Nacional de Costa Rica. Comunicación personal. Cartago, Costa Rica.
} 
La rentabilidad del modelo propuesto, de acuerdo con los supuestos establecidos anteriormente, dio a entender que el proyecto es viable económicamente, ya que se podrían obtener flujos de caja netos positivos a lo largo de los diez años de evaluación (Cuadro 4); a su vez el VAN fue positivo por $\$ 31455,39$ y la TIR fue de 21,48\%. Para efectos del VAN, el productor en moneda actual, podría recuperar la inversión inicial del año 0 y obtener ganancias en los años evaluados, mientras que la TIR fue mayor a la tasa aceptable de rendimiento supuesta (14\%), lo que

Cuadro 4. Flujo de caja del modelo productivo supuesto para los años evaluados en el establecimiento de un modelo productivo de gallina ponedora con acceso a pastoreo. Cartago, Costa Rica. 2017.

Table 4. Cash flow of the production model for the years evaluated in the establishment of a production model of laying hens with access to grazing. Cartago, Costa Rica. 2017.

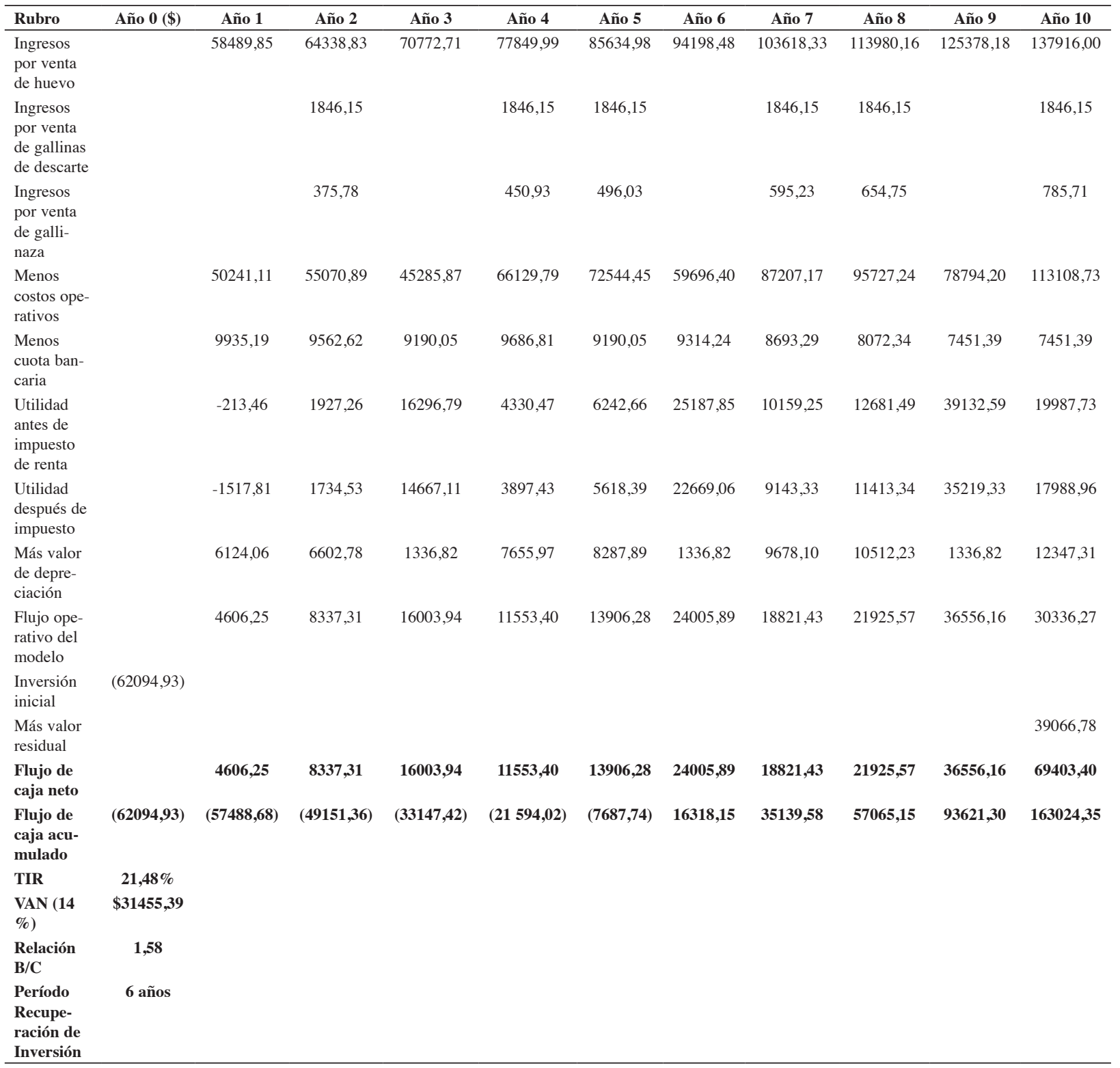


haría al proyecto rentable. Los ingresos y egresos a través de los años proyectados, fueron considerados al valor actual, resultando en una relación B/C positiva y mayor a 1. Lo que indicó que los beneficios (ingresos) superarían los costos e incidirían en ganancias.

\section{Análisis de sensibilidad del modelo de producción propuesto}

Al variar el porcentaje de postura, el precio del kilogramo de huevo y el precio del kilogramo de alimento balanceado en el análisis de sensibilidad del modelo propuesto se generaron cambios en los indicadores económicos evaluados (VAN y TIR) (Cuadro 5), donde se notó, que el escenario pesimista de los indicadores provocaría la no factibilidad de la producción de huevos con acceso a pastoreo.

Cuadro 5. Respuesta del valor actual neto (VAN) y tasa interna de retorno (TIR), a cambios en el porcentaje de postura, precio de venta del kilogramo de huevo y compra del alimento balanceado, en el establecimiento de un sistema productivo de gallina ponedora con acceso a pastoreo. Cartago, Costa Rica. 2017.

Table 5. Response of the net present value (VAN) and internal rate of return (TIR), to posture percentage, kilogram egg sale price and purchase of balanced feed, in the establishment of a productive system of laying hen with access to grazing. Cartago, Costa Rica. 2017.

\begin{tabular}{lccc}
\hline & & Escenario & \\
\cline { 2 - 4 } Indicadores & Pesimista & Normal & Optimista \\
\hline Porcentaje de postura (\%) & 73 & 84 & 95 \\
Precio kg de huevo (\$) & 2,94 & 3,27 & 3,60 \\
Precio kg de alimento balanceado (\$) & 0,47 & 0,43 & 0,39 \\
VAN (\$) & $-67796,84$ & 67796,84 & 141252,36 \\
TIR \% & $-7,44$ & 21,48 & 45,94 \\
\hline
\end{tabular}

En el caso del porcentaje de postura, los valores utilizados en el análisis de sensibilidad correspondieron a los límites inferior, promedio y superior que indica la guía de manejo de la línea genética Isa Brown, mientras que el precio de venta del huevo y de compra del alimento balanceado los afectó por $\pm 5 \%$. Bajo estas mismas circunstancias, los valores máximos y mínimos de los indicadores sensibilizados que podría soportar el modelo propuesto, bajo los escenarios pesimista y normal, para que este sea viable, manteniendo las demás variables constantes, se muestran en el Cuadro 6.

Cuadro 6. Valores máximos y mínimos del porcentaje de postura, precio de venta del kilogramo de huevo y el precio de compra del alimento balanceado para que el establecimiento de un sistema productivo de gallina ponedora con acceso a pastoreo sea viable. Cartago, Costa Rica. 2017.

Table 6. Maximum and minimum values of the posture percentage, egg kilogram sale price and purchase price of balanced feed for the establishment of a viable productive system of laying hens with access to grazing. Cartago, Costa Rica. 2017.

\begin{tabular}{lcc}
\hline & & Escenario \\
Indicadores & Pesimista & Normal \\
\hline Porcentaje de postura mínimo (\%) & 90 & 78 \\
Precio kg de huevo mínimo (\$) & 3,60 & 3,03 \\
Precio máximo kg de alimento balanceado $(\$)$ & 0,24 & 0,54 \\
\hline
\end{tabular}




\section{Discusión}

Los sistemas con acceso a pastoreo evaluados utilizaron líneas genéticas con alto potencial de producción de huevos (Cuadro 1) (Muñoz \& Vellojin, 2002), excepto las granjas 1 y 6, las cuales utilizaban un cruce de la línea Plymouth Rock Barrada (Línea 60) y Rhode Island Red, conocido por su rusticidad y desempeño productivo en niveles de nutrición subóptimos, y su uso para producción de huevos y carne (Araya-Vargas \& Chacón-Alfaro, 2011). Para seleccionar una línea genética para un sistema de producción es importante considerar el bienestar, sanidad, productividad y el índice de crecimiento de las aves (Organización Mundial de Sanidad Animal, 2015).

En el caso de las densidades encontradas (aves $\mathrm{m}^{-2}$ ), la producción de aves con acceso a pastoreo utilizados en Costa Rica, presentaron similitud con los sistemas Free range (Salatin, 1996) y Label Rouge (Plamondon, 2003), donde se manejan densidades entre ocho y nueve aves $\mathrm{m}^{-2}$ en galera y de un ave $\mathrm{m}^{-2}$ al aire libre. No así, con otros sistemas de producción con acceso a pastoreo como el orgánico (Berg, 2001), el ecológico (Soler \& Fonseca, 2011) y el aviario (Heerkens et al., 2015), donde se exige un área de $4 \mathrm{~m}^{2}$ por ave al aire libre, más limitaciones en el uso de promotores de crecimiento, entre otros. Estas formas de manejo, se asocian a la posibilidad que tiene el ave a presentar niveles altos de comportamientos de forma natural, como la acción de volar, correr, caminar, aletear, estirarse, acicalarse, ponerse de pie, sentarse, pastorear, baños de arena, anidación, uso de perchas, canibalismo y picaje; y en menor medida, las estereotipias y agresión social (Lay et al., 2011).

Al valorar la nutrición de las aves en sistemas con acceso a pastoreo, se debe considerar que el alimento balanceado debe aportar el $100 \%$ de los requerimientos nutricionales, ya que por las características digestivas del ave, el aporte de nutrimentos obtenidos del forraje pastoreado e insectos se considere como adicional a los requerimientos. En el caso, que se considere un aporte por parte del material pastoreado, este no debe superar el $5 \%$ de lo requerido, para no generar deficiencias alimenticias (Crawley, 2015). Por tal motivo, si se observa un comportamiento voraz en el ave por el forraje (Sandilans, 2016), como se pudo observar en la granja 1 del presente estudio, asociado a un proceso de subalimentación o hambre crónica, lo cual podría provocar problemas de agresión social (canibalismo y picaje) (Ricaurte-Galindo, 2005) y consecuentemente, afectar su bienestar y producir pérdidas en la productividad de huevo. La uniformización de la pastura (corta) posterior a la salida de las aves, más una adecuada rotación (menor cantidad de días de rebrote), es una estrategia que potencia la utilización del forraje, debido a que, a menor días de rebrote, el contenido celular de los forrajes es mayor (varía según la especie forrajera), valor que disminuye conforme el forraje madure. Además, se recomienda que la altura ideal del pasto para ser pastoreado por las aves sea de 3 a $5 \mathrm{~cm}$ (Vargas, 2001), por encima de $10 \mathrm{~cm}$ se le dificulta a las aves rascar el suelo en busca de insectos y se producen problemas de impactación del buche (Department for Environment, Food, and Rural Affairs, 2001), las excretas no hacen contacto con el suelo y el forraje se mantiene sucio y presenta un menor contenido celular (Carballo, 2004). Otro aspecto a considerar en el manejo del cultivo del forraje en estos sistemas, es que un tercio del parque se va deteriorar, debido a que las aves se acumulan cerca de las instalaciones por protección o acceso a la sombra y a que los comportamientos de rasgar el suelo, anidación y baños de arena, se concentran en estas áreas, tal como se observó en las aves estudiadas.

El pastoreo de las aves está regulado en los sistemas alternativos en un tiempo mínimo de seis horas al día (Diario Oficial de las Comunidades Europeas, 1999; Humane Farm Animal Care, 2014), en el caso de esta investigación el $50 \%$ de los sistemas cumplieron este mínimo de horas, pero con el agravante de que, por un mal manejo de las pasturas, el suelo se encontraba descubierto y las aves no tenían material para pastorear. Por tal motivo, los sistemas evaluados presentaron oportunidades de mejora en el manejo de los parques, donde la división del área de pastoreo es prioridad, acorde a los días de rebrote que permitan alturas entre 3 y $5 \mathrm{~cm}$ en el forraje y aumentar el número de accesos a las áreas de pastoreo, donde se recomiendan $2 \mathrm{~m}$ de abertura de las puertas o salidas de las galeras disponibles por cada 1000 aves (Diario Oficial de las Comunidades Europeas, 1999) con $35 \mathrm{~cm}$ de altura y $40 \mathrm{~cm}$ de ancho como mínimo. Estas condiciones incrementan la posibilidad de que más aves 
puedan salir al pastoreo (Harlander-Matauschek et al., 2006), sin dejar de lado la ubicación de este acceso en la galera, la genética de las aves, experiencias previas en las áreas de pastoreo, la edad, la intensidad lumínica (galera y las salidas), la presencia de fractura en la quilla, aves extrañas, depredadores, la cobertura vegetal del parque y las condiciones ambientales presentes (Gebhardt-Henrich et al., 2014; Richards et al., 2011).

Los sistemas alternativos de producción de huevos en la actualidad, surgen por la preocupación de los consumidores de que las aves produzcan bajo condiciones de bienestar, donde se pueda cuantificar el impacto de las acciones humanas sobre los animales (Main et al., 2005) y de bienestar animal, donde no se presenten limitaciones de espacio por ave, haya contacto social y estimulación ambiental (Rault et al., 2016). Estos sistemas inciden en la rentabilidad y los costos de producción, donde podrían duplicarse e incrementar en $40 \%$ el precio final del huevo (Sumner et al., 2011). Sin embargo, los gastos reflejarían incrementos relacionados a las modificaciones de las instalaciones, aumento del consumo de alimento balanceado para satisfacer las necesidades energéticas de las aves por una mayor movilidad, mayor mano de obra en la recolección de los huevos, manejo del cultivo del forraje y manipulación de las aves.

La integración de los componentes en esta modalidad alternativa de producción, provocan que el $47 \%$ de los costos iniciales se relacionen con la compra, alimentación, manejo de las aves y comercialización de los huevos. A pesar de un flujo acumulado de \$62 094,93 en el año cero y los indicadores productivos y económicos utilizados, los resultados de la evaluación económica permitirían la recuperación de la inversión en seis años, generando indicadores de TIR y VAN positivos. Además, bajo el criterio del costo hundido: en la inversión de la compra del terreno, equipo, ni la construcción de la infraestructura completa, el escenario obtenido en los sistemas productivos de huevo de piso que solo necesitarían adaptaciones, se mejorarían los indicadores de TIR en 128,59 \% y de VAN en $\$ 110359,19$. Esto porque se reduciría la inversión inicial en 81 \% y la cuota bancaria (amortización e intereses) a lo largo de los años evaluados, lo cual podría incentivar la implementación de este tipo de sistemas, bajo los supuestos establecidos, siempre y cuando el espacio físico no sea la limitante a la hora de diseñar los parques o áreas de pastoreo.

\section{Conclusiones}

El manejo de las gallinas con acceso a pastoreo para la producción de huevo por parte de las granjas en Costa Rica presentaron oportunidades de mejora en el cumplimiento de los requerimientos nutricionales, prácticas de bioseguridad e implementación de un sistema de pastoreo que permita la sostenibilidad del cultivo del forraje, optimizar los recursos utilizados y el manejo de las aves.

Las granjas con producción de gallinas ponedoras con acceso a pastoreo bajo los indicadores analizados en el modelo propuesto, mostraron un VAN positivo por $\$ 31455,39$, un TIR de $21,48 \%$ y una relación B/C>1. Al sensibilizarse el modelo, el proyecto supuesto pudo soportar un porcentaje mínimo de postura del $78 \%$, un precio mínimo de venta de $\mathrm{kg}$ de huevo de $\$ 3,03$ y un precio máximo por kilogramo de alimento balanceado de $\$ 0,54$.

La mejora en los indicadores económicos bajo el criterio de costo hundido, generó el escenario para que los sistemas convencionales de producción de huevo en Costa Rica, sin limitaciones de espacio, puedan desarrollar una línea de trabajo para disminuir la cantidad de aves por metro cuadrado.

\section{Agradecimientos}

Los autores agradecen a los productores que participaron en este estudio, que de forma desinteresada permitieron recolectar información de cada sistema y generar oportunidades de mejora en el desarrollo de modelos de producción de aves con acceso a pastoreo. 


\section{Referencias}

Araya-Vargas, J., \& Chacón-Alfaro, D. (2011). Diagnóstico para la implementación de una granja de investigación y docencia en el módulo avícola de la Estación Experimental Agrícola Fabio Baudrit Moreno de la Universidad de Costa Rica [Tesis de licenciatura, Universidad de Costa Rica]. Repositorio de la Universidad de Costa Rica. http://repositorio. sibdi.ucr.ac.cr:8080/jspui/handle/123456789/2266

Axtell, R. C., \& Arends, J. J. (1990). Ecology and management of arthropod pests of poultry. Annual Review of Entomology, 35 , 101-126. http://doi.org/10.1146/annurev.en.35.010190.000533

Bell, D. \& Weaver, W. Jr. (2002). Commercial chicken meat and egg production (5 ${ }^{\text {th }}$ Ed.). Springer Science *Business Media, LLC. https://doi.org/10.1007/978-1-4615-0811-3

Berg, C. (2001). Health and welfare in organic poultry production. Acta Veterinaria Scandinavica, 95(Suppl 1), 37-45. https:// doi.org/doi:10.1186/1751-0147-43-S1-S37

Brenes, C. (2012, setiembre 2). Huevos de pastoreo ganan mercado. El Financiero. https://www.elfinancierocr.com/negocios/ huevos-de-pastoreo-ganan-mercado/XPVEKAOFGBDUXLWTVMMU757KOA/story/

Campbell, D. L. M., Hinch, G. N., Dyall, T. R., Warin, L., Little, B. A., \& Lee, C. (2017). Outdoor stocking density in freerange laying hens: radio-frequency identification on range use. Animal, 11(1), 121-130. http://doi.org/10.1017/ S1751731116001154

Carballo, C. (2004). Manual de manejo de pollos ecológicos. ZOE Tecno-Campo. http://www.zoetecnocampo.com/Documentos/ pollo_ecol/pollos.htm

Carrillo, L., Medina-Torres, L., Tapia, G., \& Guti. L. (2016). Rheological study of healthy chicken's pooled tracheobronchial secretions and its modification by mucolytics drugs. Poultry Science, 95(11), 2667-2672. http://doi.org/doi:10.3382/ ps/pew209

Crawley, K. (2015). Fulfilling $100 \%$ organic poultry diets: Roughage and foraging from the range (Technical Note 2). Organic Research Center. https://orgprints.org/36526/1/36526_Tool_29_Ok-Net-Ecofeed-fulfilling-100-poultry-diets.pdf

Chaves, R. (2011). Servicio Nacional de Salud Animal: programa nacional de salud aviar. Servicio Nacional de Salud Animal.

Department for Environment, Food, and Rural Affairs. (2001). The welfare of hens in free range systems. https://www.gov.uk/ government/publications/the-welfare-of-hens-in-free-range-systems

Dhama, K., Latheef, S., Mani, S., Samad, H., Karthik, K., Tiwari, R., Khan, R., Alagawany, M., Farag, M., Alam, G., Laudadio, V., \& Tufarelli, V. (2015). Multiple beneficial applications and modes de action of herbs in poultry health and production-A review. International Journal of Pharmacology, 11(3), 152-176. http://doi.org/doi:10.3923/ ijp.2015.152.176

Diario Oficial de las Comunidades Europeas. (1999). Directiva 1999/74/CE del Consejo de 19 de julio de 1999 por la que se establecen las normas mínimas de protección de las gallinas ponedoras. EUR-Lex. Recuperado el 22 de mayo, 2017, de https://eur-lex.europa.eu/legal-content/ES/TXT/PDF/?uri=CELEX:31999L0074\&from=EN

Gebhardt-Henrich, S., Toscano, M., \& Fröhlich, E. (2014). Use of outdoor ranges by laying hens in different sized flocks. Applied Animal Behaviour Science, 155, 74-81. http://doi.org/doi:10.1016/j.applanim.2014.03.010 
Harlander-Matauschek, A., Felsenstein, K., Niebuhr, K., \& Troxler, J. (2006). Influence of pop hole dimensions on the number of laying hens outside on the range. Journal British Poultry Science, 47(2), 131-134. http://doi. org/10.1080/00071660600610591

Heerkens, J. L., Delezie, E., Zoons, J., Kempen, I., Ampe, B., Rodenburg, T. B. \& Tuyttens, F. A. (2015). Specific characteristics of the aviary housing system affect plumage condition, mortality and production in laying hens. Poultry Science, 94(9), 2008-2017. http://doi.org/doi:10.3382/ps/pev187

Humane Farm Animal Care. (2014). Normas del HFAC para la producción de gallinas ponedoras. http://certifiedhumane.org/ wp-content/uploads/2014/05/GALLINAS-PONEDORAS.pdf

Kahrs, R. F. (1995). Principios generales de desinfección. Revue Scientifique et Technique, 14(1), 143-163. https://www.oie.int/ doc/ged/D8972.PDF

Lay, D. C., Fulton, R. M., Hester, P. Y., Karcher, D. M., Kjaer, J. B., Mench, J. A., Mullens, B. A., Newberry, R. C., Nicol, C. J. O`Sullivan, N. P. \& Porter, R. (2011). Hen welfare in different housing systems. Poultry Science, 90, 278-294. http:// doi.org/doi:10.3382/ps.2010-00962.

Lohmann Tierzuch. (2013). Management guide. Alternative systems. Management-recommendations for the rearing of pullets in deep litter, perchery and free - range systems. Doc Player. https://docplayer.net/40980709-Managementguidealternative-systems-management-recommendations-for-the-rearing-of-pullets-in-deep-litter-perchery-and-free-rangesystems.html

Main, D. C. J., Thornton, P., \& Kerr, K. (2005). Teaching animal welfare science, ethics, and law to veterinary students in the United Kingdom. Journal Veterinary Medical Education, 32(4), 505-508. http://doi.org/doi:10.3138/jvme.32.4.505

Miao, Z. H., Glatz, P. C., Ru, Y. J., Wyatt, S. K., \& Rodda, B. J. (2005). Integrating free-range hens into a wheat stubble. International Journal Poultry Science, 4(8), 526-530. http://doi.org/doi:10.3923/ijps.2005.526.530

Monge, D. (2016, octubre 4-5). Caracterización de la producción de huevo de gallina en Costa Rica [Presentación en conferencia]. En Instituto Nacional de Estadística y Censos (Ed.), Simposio Una Visión del Sector Agropecuario Basada en el CENAGRO 2014 (pp. 9-28). Instituto Nacional de Estadística y Censos. https://inec.cr/sites/default/files/ documetos-biblioteca-virtual/imgsimposio-cenagro-08122017.pdf

Muñoz, D. (2016). Gallinas libres huevos caros. El Financiero. Recuperado el 18 de mayo, 2017, de http://www.elfinanciero. com.mx/empresas/gallinas-libres-huevos-caros.html

Muñoz, J. \& Vellojin, J. (2002). Diseño y evaluación de un sistema de producción de huevos con gallinas bajo pastoreo en el trópico húmedo. Universidad Earth. http://www.fao.org/fileadmin/templates/lead/pdf/03_article03_es.pdf

Organización Mundial De Sanidad Animal. (2015). Bienestar animal y sistemas de producción de pollos de engorde. https:// www.oie.int/index.php?id=169\&L=2\&htmfile=chapitre_aw_broiler_chicken.htm

Plamondon, R. (2003). Range poultry housing. Livestock Production Guide. University of California. https://www.ucanr.edu/ sites/placernevadasmallfarms/files/102339.pdf

Rault, J. L., Hemsworth, P., \& Cronin, G. (2016). Free range hen welfare: Characterisation of “outdoor" and "indoor” hens and physical features in the range. Australian Egg Corporation Limited.https://www.australianeggs.org.au/dmsdocument/708free-range-hen-welfare-characterisation-of-outdoor-and-indoor-hens-and-physical-features-in-the-range 
Ricaurte-Galindo, S. (2005). Bioseguridad en granjas avícolas. Revista Electrónica de Veterinaria, 6(2), 1-17. https://www. redalyc.org/pdf/636/63612654015.pdf

Richards, G., Wilkins, L., Knowles, T., Booth, F., Toscano, M., Nicol, C., Brown, S., \& Booth, F. (2011). Continuous monitoring of pop hole usage by commercially housed free-range hens throughout the production cycle. Veterinary. Record, 169(13), 338. http://doi.org/10.1136/vr.d4603

Ruiz, B. (2017). Peculiaridades de la industria avícola en el 2016. Industria Avícola, 64(3), 14-34. http://www.industriaavicoladigital.com/201703/\#/16

Sandilans, V. (2016). Chicken behaviour and welfare. Online Course. University of Edimburgh. https://www.ed.ac.uk/studying/ online-learning/free-short-courses/subjects/medicine-vet/chicken-behaviour-welfare

Salatin, J. (1996). Pastured Poultry Profit. Polyface Inc, Swoope.

Sapag, N., \& Sapag, R. (2008). Preparación y evaluación de proyectos (5 ${ }^{\text {ta }}$ Ed.). McGraw-Hill Interamericana S.A.

Secretaria Ejecutiva de Planificación Sectorial Agropecuaria. (2018). Boletín estadístico agropecuario (Serie cronológica 20142017, 28 Ed.). Ministerio de Agricultura y Ganadería. http://www.sepsa.go.cr/DOCS/BEA/BEA28.pdf

Singh, M., \& Cowieson, A. J. (2013). Range use and pasture consumption in free-range poultry production. Animal Production Science, 53(11), 1202-1208. http://doi.org/10.1071/AN13199

Soler, D. M., \& Fonseca J. A. (2011). Producción sostenible de pollo de engorde y gallina ponedora campesina: revisión bibliográfica y propuesta de un modelo para pequeños productores. Revista de Investigación Agraria y Ambiental, 2(1), 29-43. https://doi.org/10.22490/21456453.914

Sumner, D. A., Gow, H., Hayes, D., Mathews, W., Norwood, B., Rosen-Molina, J., \& Thurman, W. (2011). Economic and market issues on the sustainability of egg production in the United States: Analysis of alternative production systems. Poultry Science, 90(1), 241-250. http://doi.org/10.3382/ps.2010-00822

Vargas, R. A. (2001). Producción de pollos de engorde bajo un sistema de pastoreo en el trópico húmedo de Costa Rica [Tesis de Licenciatura, no publicada]. Universidad Earth. 